\title{
Anabases
}

ANABASES Traditions et réceptions de l'Antiquité

$30 \mid 2019$

Varia

\section{Le sanctuaire gaulois de Gournay-sur-Aronde (Oise). Retour sur une découverte exceptionnelle des années 1970}

\section{Carole Quatrelivre}

\author{
(2) OpenEdition \\ Journals \\ Édition électronique \\ URL : https://journals.openedition.org/anabases/9669 \\ DOI : $10.4000 /$ anabases.9669 \\ ISSN : 2256-9421 \\ Éditeur \\ E.R.A.S.M.E.
}

\section{Édition imprimée}

Date de publication : 21 octobre 2019

Pagination : 11-30

ISSN : 1774-4296

\section{Référence électronique}

Carole Quatrelivre, «Le sanctuaire gaulois de Gournay-sur-Aronde (Oise). Retour sur une découverte exceptionnelle des années 1970 », Anabases [En ligne], 30 | 2019, mis en ligne le 21 octobre 2021, consulté le 08 novembre 2021. URL : http://journals.openedition.org/anabases/9669 ; DOI : https:// doi.org/10.4000/anabases.9669 

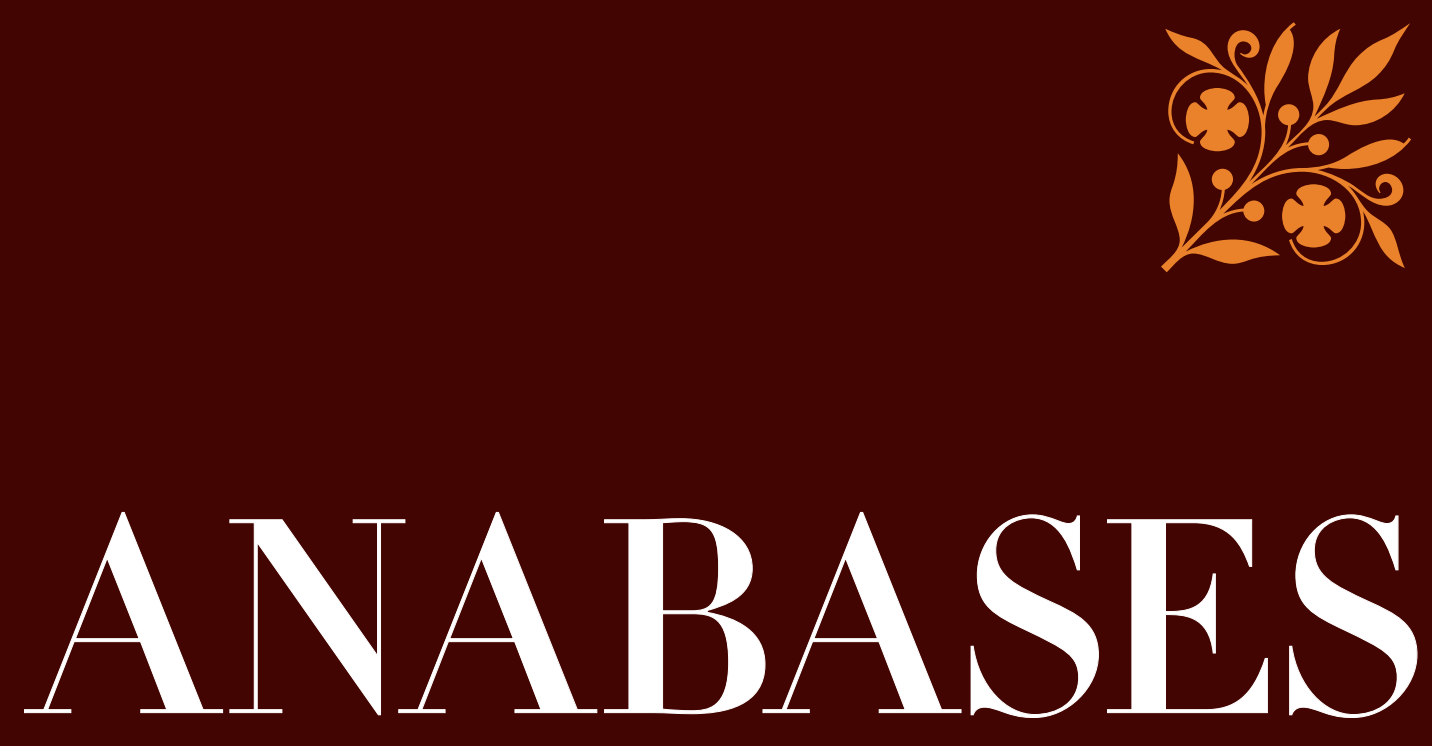

Traditions et Réceptions de l'Antiquité

\section{$\mathrm{N} \times 30$ \\ 2019}

Le sanctuaire de Gournay-sur-Aronde Le pindarisme et l'archéologie musicale Hélène en Égypte Perpétuer Ovide aux $X I V^{e}-X V I I I^{e}$ siècles II classico si fa pop Relire Marcel Detienne Freud à Pompéi 
ANABASES

Traditions et Réceptions de l'Antiquité

Revue de l'équipe de recherche E.R.A.S.M.E.

Université Toulouse-Jean Jaurès (UT2J)

Anabases dispose d'un Comité de lecture international. Chaque article envoyé à la rédaction est soumis, une fois anonymisé, à l'expertise de deux spécialistes qui rendent un rapport écrit. Les deux rapports anonymisés sont transmis à l'auteur qui tient compte des observations en vue de la publication.

\section{Comité SCIENTIFIQue}

Germaine Aujac (université Toulouse-Jean Jaurès : histoire de la géographie et des sciences antiques)

Florence Bouchet (université Toulouse-Jean Jaurès : littérature médiévale)

Hinnerk Brunns (CNRS : histoire économique et sociale ancienne et contemporaine)

Paulo Butti de Lima (université de Bari : historiographie et réception de l'Antiquité)

Luciano CANFora (université de Bari : littérature et histoire anciennes, historiographie)

Giovanna Ceserani (Stanford University : histoire intellectuelle et historiographie de la tradition classique)

Temístocles Cezar (université de Porto Alegre : historiographie moderne)

Serafina Сuомо (University of London, Birkbeck College : histoire des mathématiques et des sciences)

Paul Demont (université de Paris Sorbonne : philologie grecque et héritage classique)

Marie-Laurence Desclos (université de Grenoble II : philosophie de l'Antiquité)

Olivier Devillers (université de Bordeaux 3 - Michel-de-Montaigne : littérature et historiographie latines)

Andrea Giardina (Istituto italiano di scienze umane : histoire du monde romain et de ses réceptions)

Ève Gran-Aymerich (AIBL : histoire de l'archéologie et des transferts culturels)

François Hartog (eHess : historiographie ancienne et moderne)

Geneviève Hoffmann (université de Picardie : histoire des mondes grecs)

Christian JACOB (CNRS/EHESS : histoire comparée et épistémologie des savoirs)

Suzanne Marchand (Louisiana State University : histoire du classicisme et de l'orientalisme)

Wilfried NIPPEL (Humboldt Universität Berlin : histoire et historiographie de l'Antiquité)

Sylvie Pitria (université de Paris I-Panthéon Sorbonne : histoire et historiographie du monde romain)

Stéphane Ratтi (université de Franche-Comté - Besançon : philologie et héritage latin)

Comité de RÉdACtion

Clément Bertau-Courbières, Corinne Bonnet, Laurent bricault, Clément Bur, Adeline Grand-Clément, Anne-Hélène Klinger-Dollé, Véronique Krings, Thibaud Lanfranchi, Pascal Payen, Grégory Reimond, Sarah Rey, Catherine Valenti, Noémie VillacèQue

ÉditeUr RESPONSABLE

Clément Bur

Éditrice ADJOINTE

Catherine VALENTI

Sites Web

http://plh.univ-tlse2.fr

Revues.org : http://anabases.revues.org

Aвonnement et vente aU numéro

Éditions De Boccard - 4, rue de Lanneau - 75005 Paris

info@deboccard.com - www.deboccard.com

Tél. : 0033/(0)143260037 - Fax : 0033/(0)143548583 




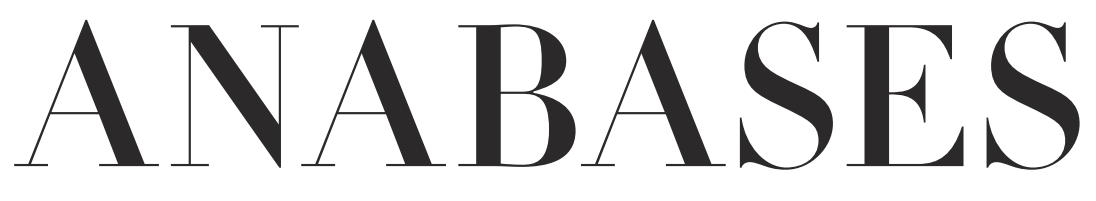

Traditions et Réceptions de l'Antiquité

$$
\begin{aligned}
& N \circ 30 \\
& 2019
\end{aligned}
$$

\section{E.R.A.S.M.E.}

Université Toulouse - Jean Jaurès 



\section{Sommaire}

ํo30 - 2019

\section{Historiographie et identités culturelles}

\section{Carole Quatrelivre}

Le sanctuaire gaulois de Gournay-sur-Aronde (Oise).

Retour sur une découverte exceptionnelle des années I970 . . . . . . . . II

\section{Traditions du patrimoine antique}

Christophe Corbier

Le pindarisme et l'archéologie musicale : style, valeur et authenticité

de la première Pythique à l'époque moderne . . . . . . . . . . . . . . .

Arnaud AmiLIEN

Hélène en Égypte : Hérodote en dialogue avec l’épopée . . . . . . . . . .

\section{Archéologie des savoirs}

Sébastien Cazalas

Au jardin des exempla. Rhétorique et stratégie de l'exemplum antique

dans l'œuvre politique de Jean Juvénal des Ursins (I388-ı473) . . . . . . 7 7

Dossier - Perpétuer Ovide : aspects moraux, éditoriaux,

linguistiques et culturels $\left(\mathrm{XIV}^{\mathrm{e}}-\mathrm{XVIII}^{\mathrm{e}} \mathrm{s}\right.$.)

Francesca Dell'Oro

Introduction $\ldots \ldots \ldots \ldots$. . . . . . . . . . . . . . 89

Hélène Casanova-Robin

L’audace châtiée : Phaéton, Actéon et Icare dans la tradition latine jusqu'à la Renaissance, tours et détours d'un symbolisme . . . . . . . . 9 93 
Dylan Bovet

Le commentaire latin des Métamorphoses d'Ovide : pratiques humanistes et évolutions de Regius-Micyllus (I543) à Burmann-Heinsius (I727) . . . . III

Martine FurNo

Ovide en classe, ou un auteur en éclats . . . . . . . . . . . . . I27

Basil NELIS

D’un Ovide chrétien à un Ovide burlesque, du Moyen Âge au Grand Siècle : continuités et changements dans la traduction et dans l'illustration des Métamorphoses perçus à travers deux éditions du xvII ${ }^{\mathrm{e}}$ siècle . . . . . . . I I43

Olivier ThÉvenAz

Épilogue ....................... I6

\section{Actualités et débats}

Tiphaine-Annabelle BEsnard

2019 : l'année pop des musées d'archéologie. Retour sur l'exposition

romaine Il classico sifa pop. Di scavi, copie e altripasticci . . . . . . . . . . I I7I

\section{Lire, relire la bibliothèque des sciences de l'Antiquité}

Jean-Pierre Albert

Le premier Detienne : une relecture de

“ La notion mythique d"A $\lambda \hat{n} \theta \varepsilon 1 \alpha$ » $\left(R E G\right.$, I96o, p. 27-35) . . . . . . . . . . $\quad{ }_{779}$

Marcel Detienne

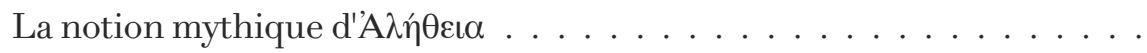

\section{Ateliers de l'histoire}

Antiquités numériques (coordonné par Elodie Guillon) ( $n^{\circ} 1$ )

Élodie GuILlon

Introduction

Jaime Alvar

Le projet EPIDI : Epítetos divinos.

Experiencia religiosa y relaciones de poder en Hispania . . . . . . . . . . . I98 Les mots de l'Antiquité (coordonné par Magali Soulatges) ( $n^{\circ} 10$ )

Jack Thомаs

L’Antiquité dans les toponymes de l'État de New York . . . . . . . . . . . 202

Actualité du théâtre (coordonné par Malika Bastin-Hammou) ( $\left.n^{\circ} 4\right)$

Mathieu FERrand

“ Avons-nous perdu le Soleil ? / Ou l'avons-nous chassé ? » 
Thyeste de Sénèque, traduit par Florence Dupont.

Mise en scène de Thomas Jolly (Avignon, 20I8) . . . . . . . . . . . . . 2 2I4

Voyages et Voyageurs (coordonné par Véronique Krings) ( $n^{\circ} 11$ )

Claude AzIzA

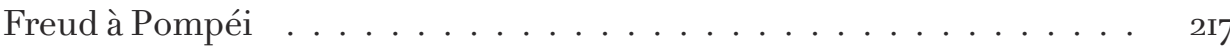

\section{Comptes rendus}

Lucile Arnoux-Farnoux et Polina Kosmadaki (dir.)

Le double voyage : Paris-Athènes (Igrg-Ig39) (Catherine Valenti) . . . . . . 225

Sandra Boehringer et Daniele Lorenzini (dir.)

Foucault, la sexualité, l'Antiquité (Jan Nelis) . . . . . . . . . . . . . . . . 226

Corinne Bonnet, Nicole Belayche, Marlène Albert Llorca,

Alexis Avdeeff, Francesco Massa, Iwo Slobodzianex (dir.)

Puissances divines à l'épreuve du comparatisme. Constructions,

variations et réseaux relationnels (Geneviève Hoffmann) . . . . . . . . . . 228

Shane ButLer (éd.)

Deep Classics, Rethinking Classical Reception (Jan Nelis) . . . . . . . . . . 23o

Zeynep ÇELIK

About Antiquities: Politics of Archaeology in the Ottoman Empire (Jorge Elices Ocón) . . . . . . . . . . . . . . . . . 231

Xavier Deru et Germaine Leman-Delerive (éd),

Franz Cumont, Comment la Belgique fut romanisée (Vivien Barrière) . . . 234

Olivier Devillers, Breno Battistin Sebastiani (éd.)

Sources et modèles des historiens anciens (Arnaud Saura-Ziegelmeyer) . . $\quad 235$

Mara Fazio, Pierre Frantz et Vincenzo De Santis (dir.)

Les Arts du spectacle et la référence antique dans le théâtre

européen (I760-I830) (Arnaud Saura-Ziegelmeyer) . . . . . . . . . . . . . . 237

Jérémy Guedu et Barbara Meazzi (dir.)

La culture fasciste entre latinité et méditerranéité (I880-1940), in Cahiers de la Méditerranée 95, (Andrea Avalli) . . . . . . . . . . . . . . . 239

Marie-Laurence HAAck (éd.), avec la collaboration de Martin MiLler, Les Étrusques au temps du fascisme et du nazisme (Jan Nelis) . . . . . . . . . 24I

Arlene Holmes-Henderson, Steven Hunt et Mai Musié (éd.)

Forward with Classics. Classical Languages in Schools and Communities

(Charlotte Tournier) . . . . . . . . . . . . . . . . . . . . . . .

Daniel Jew, Robin Osborne et Michael Scotт (éd.)

M. I. Finley. An Ancient Historian and his Impact (Hinnerk Bruhns) . . . . $\quad 244$ 
Kostas Kalimtzis,

An inquiry into the philosophical concept of scholê.

Leisure as a Political End (Florent Rouzade) . . . . . . . . . . . . . . . . . 246

Anne-Hélène KLINGER-Dollé

Le De sensu de Charles de Bovelles. Conception philosophique

des sens et figuration de la pensée. Suivi du texte latin du De sensu,

traduit et annoté (Laure Hermand-Schebat) . . . . . . . . . . . . . 248

Egidia Occhipinti

The Hellenica Oxyrhynchia and Historiography:

New Research Perspectives (Anne de Cremoux) . . . . . . . . . . . . . . . . 249

Laurent OLIVIER (dir.)

La mémoire et le temps. L'ouvre transdisciplinaire

d'Henri Hubert (I872-I927) (Sarah Rey) . . . . . . . . . . . . . . . 25I

Gabriella Pinonti et Corinne Bonnet (dir.),

Les dieux d'Homère. Polythéisme et poésie en Grèce ancienne,

Kernos, Supplément 3r. (Sandya Sistac) . . . . . . . . . . . . . . . 253

Rabun TAYLoR, Katherine W. Rinne et S. Kostof

Rome. An Urban History from Antiquity to the Present

(Cyrielle Landrea) . . . . . . . . . . . . . . . . . . . 255

Wyger Velema et Arthur Weststeisn (éd.)

Ancient Models in the Early Modern Republican Imagination

(Paulo Butti de Lima) . . . . . . . . . . . . . . . . . . . 256

Philip WaLsh (éd.)

Brill's Companion to the Reception of Aristophanes

(Malika Bastin-Hammou) . . . . . . . . . . . . . . .

Richard WARren

Art Nouveau and the Classical Tradition (Lucien Calvié) . . . . . . . . . 26o

Jesse Weiner, Benjamin Eldon Stevens et Brett M. Rogers (éd.)

Frankenstein and Its Classics. The Modern Prometheus

from Antiquity to Science Fiction (Mathieu Scapin) . . . . . . . . . . . . . 26r

Nigel G. WiLson

From Byzantium to Italy. Greek Studies in the Italian Renaissance,

deuxième édition (Luigi-Alberto Sanchi) . . . . . . . . . . . . . . 262

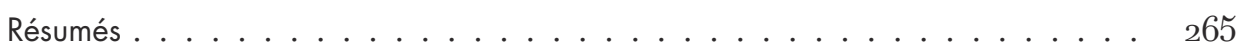

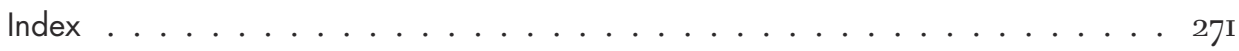




\section{Historiographie et identités culturelles}





\section{Le sanctuaire gaulois de Gournay-sur- Aronde (Oise). Retour sur une découverte exceptionnelle des années 1970}

Carole Quatrelivre

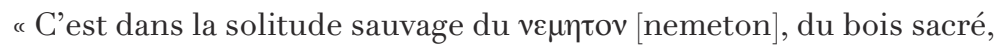
que la tribu celtique rencontre ses dieux, et son monde mythique est une forêt sacrée, sans routes et sans limites, toute peuplée de puissances mystiques $(\ldots)^{1}$. ’

Tel est le tableau pour le moins romantique que brosse M.-L. Sjoestedt des Celtes et de leurs pratiques religieuses, dans son ouvrage de 1940. Cette description, héritée du xIx ${ }^{\mathrm{e}}$ siècle, replace les Celtes dans un cadre naturel vierge, un environnement sur lequel ces hommes n’auraient laissé aucune trace, sinon dans la mémoire des Anciens. Cette perception du Celte « bon sauvage », dont l'oralité de la tradition allait nécessairement de pair avec des cultes naturistes, sans matérialité pérenne, subsiste encore jusque dans les années 1970 en France. En 1977, la mise au jour du sanctuaire gaulois de Gournay-sur-Aronde bouleverse profondément ces idées reçues ${ }^{2}$.

Implanté dans la vallée de l'Aronde, un affluent de l'Oise, le site a révélé un lieu de culte daté entre le $\mathrm{Iv}^{\mathrm{e}}$ siècle av. J.-C. et le $\mathrm{I}^{\mathrm{er}}$ siècle ap. J.-C. (fig. 1). Bénéficiant

1 M.-L. Sjoestedt, Dieux et héros des Celtes, Paris, Presses universitaires de France, collection Mythes et religions, 1940, p. 123.

2 Les sociétés celtiques s'établissent entre le second quart du Ve s. av. J.-C. et le dernier tiers du I ${ }^{\text {er }}$. av. J.-C. en Europe tempérée; elles sont également dites " gauloises » ou “ laténiennes » d'après le site éponyme de La Tène, en Suisse. 


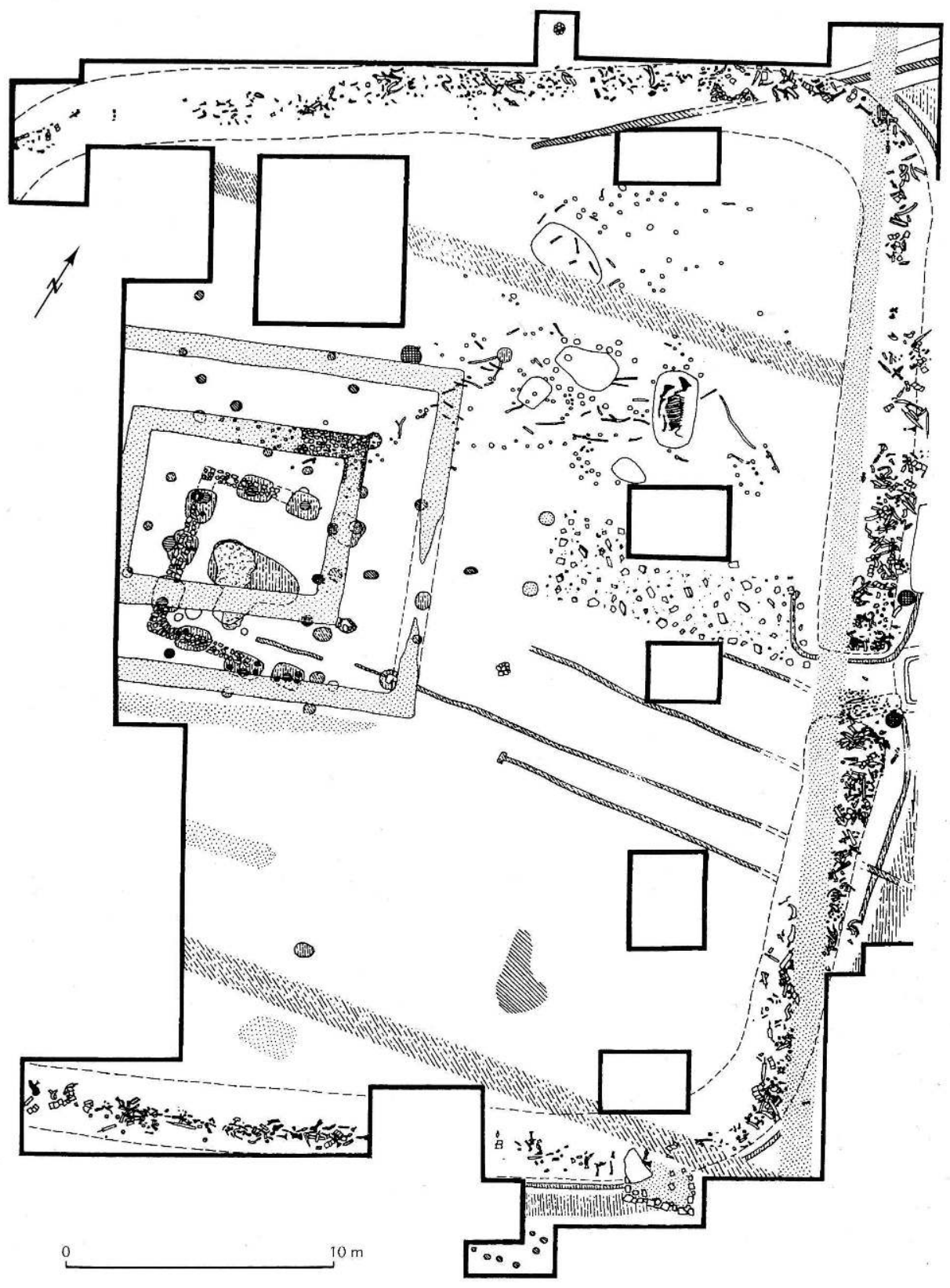

Fig. 1 : Plan général du sanctuaire gaulois de Gournay-sur-Aronde (Oise). D'après J.-L. Brunaux et al., 2003, p. 17. 
du développement institutionnel de l'archéologie dans les années 1970, cette découverte est la première en son genre à faire l'unanimité de la communauté scientifique européenne. Elle est rapidement érigée en modèle, qui perdure jusqu'à ce jour. La recherche sur la religion celtique se mue en une véritable approche archéologique des pratiques rituelles à travers l'observation minutieuse des artefacts. Cette évolution méthodologique a eu d'importantes répercussions par la suite sur l'archéologie et l'histoire des religions classiques, qui adopteront la démarche protohistorienne.

\section{Le dynamisme de l'archéologie picarde dans les années 1970}

En Picardie, l'institutionnalisation de l'archéologie se renforce pendant les années 1970 pour répondre à l'accélération des opérations d'aménagement du territoire, et tout particulièrement la multiplication des carrières et des sablières ${ }^{3}$. Rattachée jusque-là au Nord-Pas-de-Calais, la Direction des Antiquités de Picardie devient autonome en 1972 sous la direction de J.-M. Desbordes pour la circonscription historique, et de R. Agache pour la circonscription préhistorique.

Des associations et groupes de recherches apparaissent localement comme à Creil, Pierrefonds et Noyons ${ }^{4}$, héritiers des sociétés savantes du xix ${ }^{\mathrm{e}}$ siècle. Ils prennent de l'ampleur dans les années 1970, avec la nomination fortuite de B. Soudsky à l'Université Paris I. Sensible au potentiel de la vallée de l'Aisne et souhaitant créer un chantier-école, ce dernier fonde en 1973 une équipe de recherches, l'URA $12 \mathrm{du}$ CNRS, ainsi que le Centre de recherches protohistoriques de Paris I qui lui est rattaché5. Cette équipe propose un programme de sauvetage aux autorités, afin de faire face à la construction d'un canal de grand gabarit dans l'Aisne. Le Conseil Général et les services extérieurs de l'État l'approuvent et lui allouent d'importants crédits annuels (100 000 francs). En 1974, une commission scientifique est nommée par décret ministériel pour encadrer les activités du groupe de recherche ${ }^{6}$, qui se poursuivront jusqu'en 2004.

3 Collectif, Vallée de l'Aisne. Cinq années de fouilles protohistoriques, Amiens, Revue archéologique de Picardie, numéro spécial, 1982, préface.

4 J.-C. Blanchet, "Histoire et avenir de la recherche archéologique dans la région de Compiègne ", in Collectif, Archéologie de la Vallée de l'Oise. Compiègne et sa région depuis les origines, Exposition au Centre culturel de Compiègne (Oise), 17 janvier 23 février 1991. Compiègne, CRAVO, 1991, p. 13.

5 E. Bellon, A. Montagne-Bôrras, “Les archives de l'équipe 'Protohistoire européenne, de la sédentarisation à l'État, (Maison René Ginouvès, archéologie et ethnologie) ", in Les Nouvelles de l’Archéologie [en ligne], n¹10, 2007. [Page consultée le 13/11/2017] https://nda.revues.org/205.

6 Collectif, Vallée de l’Aisne, préface. 
En favorisant l'élaboration d'un programme scientifique, les élus ont fait le choix d'une véritable « stratégie de sauvetage ${ }^{7}$ » du patrimoine. Grâce à un inventaire et une cartographie actualisés des sites, une approche prédictive des occupations archéologiques dans le territoire devient possible. Il s'agit de proposer, en amont des projets de constructions, une estimation plus ou moins précise du potentiel archéologique du secteur aménagé. Cette démarche demeure encore aujourd'hui en vigueur, à travers les prescriptions délivrées par les Services régionaux de l'archéologie (même si les moyens, les méthodes et la réglementation ont évolué depuis).

Toujours en 1974, une autre association naît d'un regroupement d'archéologues : le Centre de Recherches Archéologiques de la moyenne Vallée de l'Oise (CRAVO), dont le premier président est J.-C. Blanchet. Il tisse immédiatement des liens avec le Musée Antoine Vivenel, alors placé sous la direction de C. Lapointe, en y installant son siège social. Au moment de sa fondation, le CRAVO fait porter ses recherches sur « l'évolution des sociétés humaines de la Préhistoire et de la Protohistoire dans la moyenne vallée de l'Oise ${ }^{8}$. »

Il s'agit alors de mettre en place une collaboration entre les archéologues locaux par le biais de réunions mensuelles, mais aussi de mutualiser le matériel nécessaire aux fouilles et aux études de mobilier archéologique. Un premier article parait dans la Revue arcgéologique de l'Oise, fait acte de manifeste. La vocation éducative du CRAVO y est mise en exergue, travaillant en collaboration avec le Centre de Documentation Pédagogique. Enfin, l'association s'implique dans le montage d'une exposition au Musée Antoine Vivenel, à l'occasion du IX Congrès international des sciences préhistoriques et protohistoriques. Intitulée « Les premiers Agriculteurs et les Âges des Métaux en Picardie », elle est ouverte du 19 juin au 31 décembre $1976^{\circ}$. Plus tard, et jusqu'à aujourd'hui, le CRAVO devient un véritable réseau au cœur de la recherche archéologique picarde.

Avec la nécessité de diffuser les résultats toujours plus abondants de l'archéologie régionale, deux revues spécialisées voient le jour dans ces années : la Revue archéologique de l'Oise en 1971, et les Cahiers archéologiques de Picardie en 1974. Ces organes de publication sont la condition sine qua non d'une recherche scientifique rigoureuse, ouverte sur le public et sont vecteurs de reconnaissance des travaux régionaux à l'échelle nationale et internationale. Les deux revues

7 G. Bailloud et al., "L'archéologie dans la vallée de l’Aisne », in Collectif, Vallée de l'Aisne, p. 11.

8 Collectif, “ Le centre de recherches et d'études archéologiques de la moyenne vallée de l'Oise », in Revue archéologique de l'Oise, n`5, 1975, p. 5.

9 Collectif, Les premiers Agriculteurs et les Âges des Métaux en Picardie, Exposition au Musée Antoine Vivenel de Compiègne (Oise), 19 juin - 31 décembre 1976, Revue Archéologique de l'Oise, nº, 1976, p. 2-3. 
fusionnent en 1982 sous le nom de Revue archéologique de Picardie ${ }^{10}$, encore éditée aujourd'hui.

Les années 1970 sont donc synonymes de développement institutionnel et légal pour l'archéologie, doublé d'un accroissement des actions locales. Si la découverte de Gournay-sur-Aronde s'inscrit en-dehors des circuits académiques conventionnels, ce contexte florissant de l'archéologie en région picarde a pu favoriser la mobilisation des acteurs du milieu et amplifier de fait son impact sur la recherche.

\section{Histoire de la découverte" 1}

\section{Une découverte fortuite}

La découverte de Gournay-sur-Aronde est liée à l'histoire locale d'un groupe d'amis. Certains étaient camarades de classe depuis le lycée ou l'Université Technologique de Compiègne, et partageaient leur passion pour l'archéologie : J.-L. Brunaux, P. Méniel, P. Marquis et P. Fluzin. Bénévoles depuis leur enfance sur divers chantiers de fouilles, ils rejoignent le CRAVO dès sa création. Grâce à ce réseau nouvellement institué, quelques premiers travaux communs se mettent en place. C'est ainsi que J.-L. Brunaux et P. Marquis conduisent une fouille ensemble à Verberie en octobre $1975^{12}$.

Au cours d'une prospection pédestre dans les environs de son village natal de Gournay-sur-Aronde, J.-L. Brunaux repère le 7 avril 1975 sur le versant ouest de la vallée de l'Aronde des céramiques brisées et étalées dans les champs par le labour. Après autorisation de la Direction des Antiquités, et grâce à la collaboration de J.-C. Blanchet et du CRAVO, il procède de mai à juin à la fouille d'un établissement gallo-romain et d'une structure pré-romaine. Celle-ci, creusée dans le limon, de forme quadrangulaire, renfermait un clou, un couteau en fer, quelques rares os de mouton et de porc ainsi qu'une série de vingt à vingt-cinq vases en céramique, disposés volontairement par taille et par type ${ }^{13}$.

10 Anonyme, "Historique », in Revue archéologique de Picardie. [Page consultée le 12/11/2017]. https://www.revue-archeologique-picardie.fr/content/6-historique.

1 Un certain nombre d'informations inédites nous ont été transmises oralement, grâce à J.-L. Brunaux, P. Méniel, P. Marquis, P. Fluzin, F. Masse, P. Gardin et F. Poplin. Qu'ils en soient vivement remerciés ici.

12 J.-L. Brunaux, P. Marquis, “Une fosse de la Tène IA à Verberie (Oise) ", in Revue archéologique de l'Oise, n6, 1975, p. 11-17.

13 J.-L. Brunaux, “Fouille de sauvetage à Gournay-sur-Aronde (Oise) ", in Revue archéologique de l'Oise, nº, 1975, p. 27-31. 
Au vu de la composition de l'assemblage d'objets et de la singularité de leur disposition, la structure est interprétée comme la sépulture à incinération d'un individu gaulois de la fin du $\mathrm{Iv}^{\mathrm{e}}$ siècle ou du début du $\mathrm{II}^{\mathrm{e}}$ siècle av. J.-C. Une restitution de cette tombe est présentée par la suite au public, dans le cadre de l'exposition du Musée Antoine Vivenel de 1976, montée en collaboration avec le CRAVO (cf. 1. $)^{14}$.

Entre 1976 et 1977, une succession de découvertes métalliques (un fer de lance et des fragments de lame d'épée) à une cinquantaine de mètres du secteur initial mettent les étudiants sur la piste d'une nécropole gauloise ${ }^{15}$. Avec l'accord du propriétaire, M. Destinay, et l'autorisation de la Direction des Antiquités, l'équipe rapidement constituée (en une semaine selon les dires de J.-L. Brunaux) commence ses travaux en mars 1977 (fig. 2). Pourtant, dès l'ouverture du chantier, les fouilleurs sont confrontés à un certain nombre d'obstacles tels que la nature

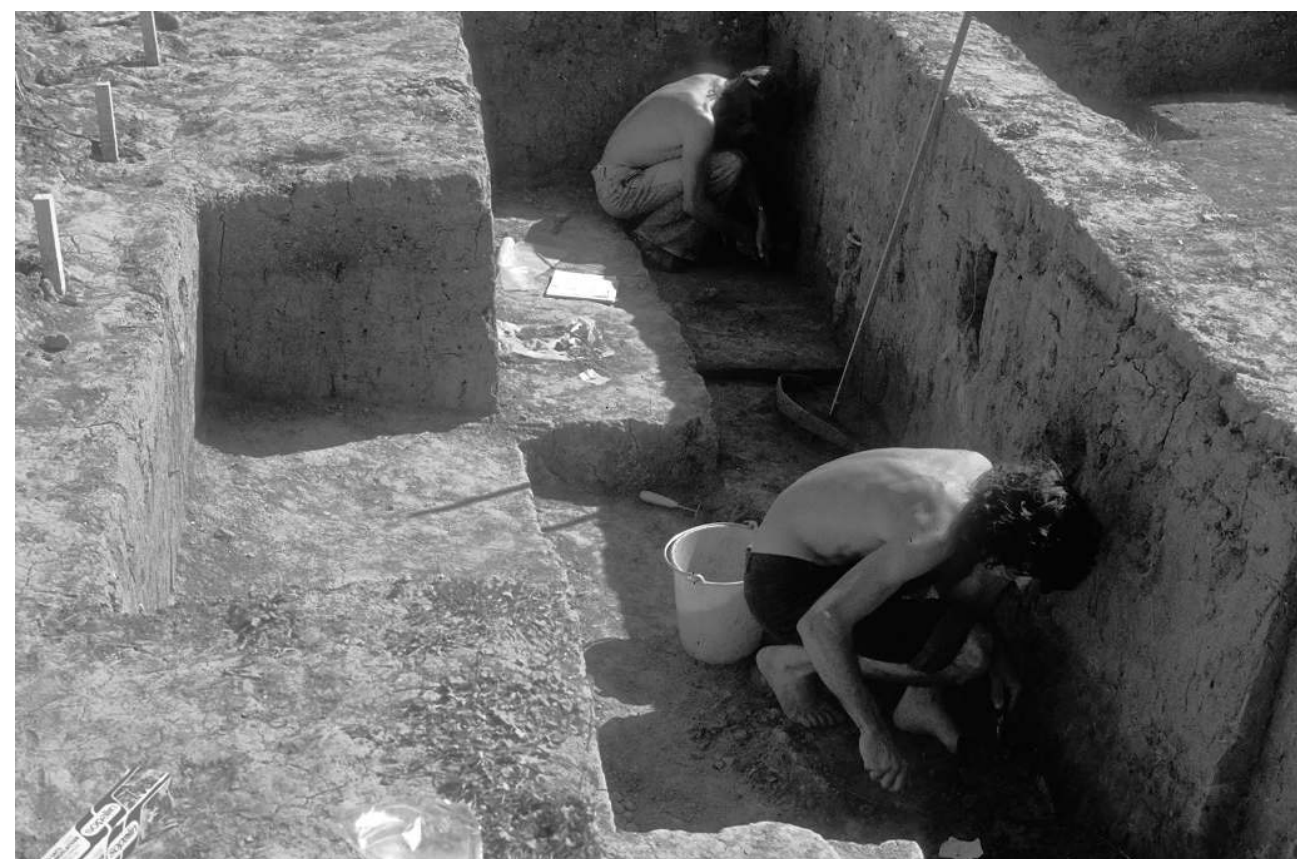

Fig. 2. J.-L. Brunaux et P. Marquis fouillant le fossé du sanctuaire. Cl. J.-L. Brunaux.

14 Collectif, Les premiers Agriculteurs.

15 J.-L. Brunaux, Fouille de sauvetage d'un enclos rituel de La Tène à Gournay-sur-Aronde (Oise), Rapport de fouille de sauvetage, Direction des Antiquités de Picardie, 1978, non paginé. 
du terrain, la complexité des structures anciennes et le manque de moyens, les amenant à adapter leurs méthodes.

\section{Une fouille complexe}

Si les fouilleurs possédaient déjà une expérience de terrain, en particulier des carrières locales, la nature des sols complique leurs travaux. En effet, le limon très homogène du site de Gournay-sur-Aronde a pour conséquence une lecture difficile des limites des structures creuses. Il n'existe pas de contraste visuel entre le limon géologique et le limon remanié par l'homme. L'équipe tente alors diverses méthodes pour faire ressortir les trous de poteaux, fosses et fossés, comme râper la surface avec une pelle aiguisée ou réaliser des empreintes au latex des coupes stratigraphiques, mais sans résultat probant. Autre contrainte : ces sols rendent également difficile tout prélèvement de terre, en vue d'analyses des restes organiques ${ }^{16}$.

Ensuite, la densité et la disposition complexe des objets dans le fossé d'enclos du sanctuaire forcent les fouilleurs à adopter une stratégie particulière (fig. 3). Ils s'inspirent de la méthode d'A. Leroi-Gourhan, développée sur le site paléolithique de Pincevent (Seine-et-Marne). Celle-ci accorde une grande importance à la documentation photographique et prône une fouille par niveaux horizontaux ${ }^{17}$. Par conséquent, à Gournay-sur-Aronde, le dégagement se fait par passes manuelles de dix centimètres de profondeur, s'appuyant sur la hauteur moyenne d'un umbo de bouclier (renfort central métallique) (fig. 4) ${ }^{18}$. Chaque niveau horizontal, numéroté de un à quinze, fait l'objet d'un relevé dessiné très précis. Par contre, la documentation photographique, composée d'au moins trois films de photographies argentiques, a été complètement perdue à la suite d'erreurs de manipulation. Une fois documentés, les objets sont prélevés, parfois par lots tant ils sont imbriqués.

Enfin, aucune subvention n'est allouée aux fouilles et à la gestion du mobilier, ce qui implique notamment l'absence d'interventions mécanisées. Un tel manque de moyens induit également une protection diminuée du site. Le chantier reste ouvert durant les trois quarts de l'année, avec des effectifs souvent très réduits P. Marquis, par exemple, se rappelle être resté seul un mois sur le terrain. Le site est victime de son succès et fait ainsi l'objet de pillages, les malfrats emportant un certain nombre d'armes qui dépassaient des coupes pratiquées dans le fossé d'enclos.

16 J.-L. Brunaux, Rapport de 1977.

17 M. Groenen, Pour une histoire de la préhistoire: le paléolithique, Grenoble, éditions J. Millon, 1994, p. 119.

18 J.-L. Brunaux, Rapport de 1977. 


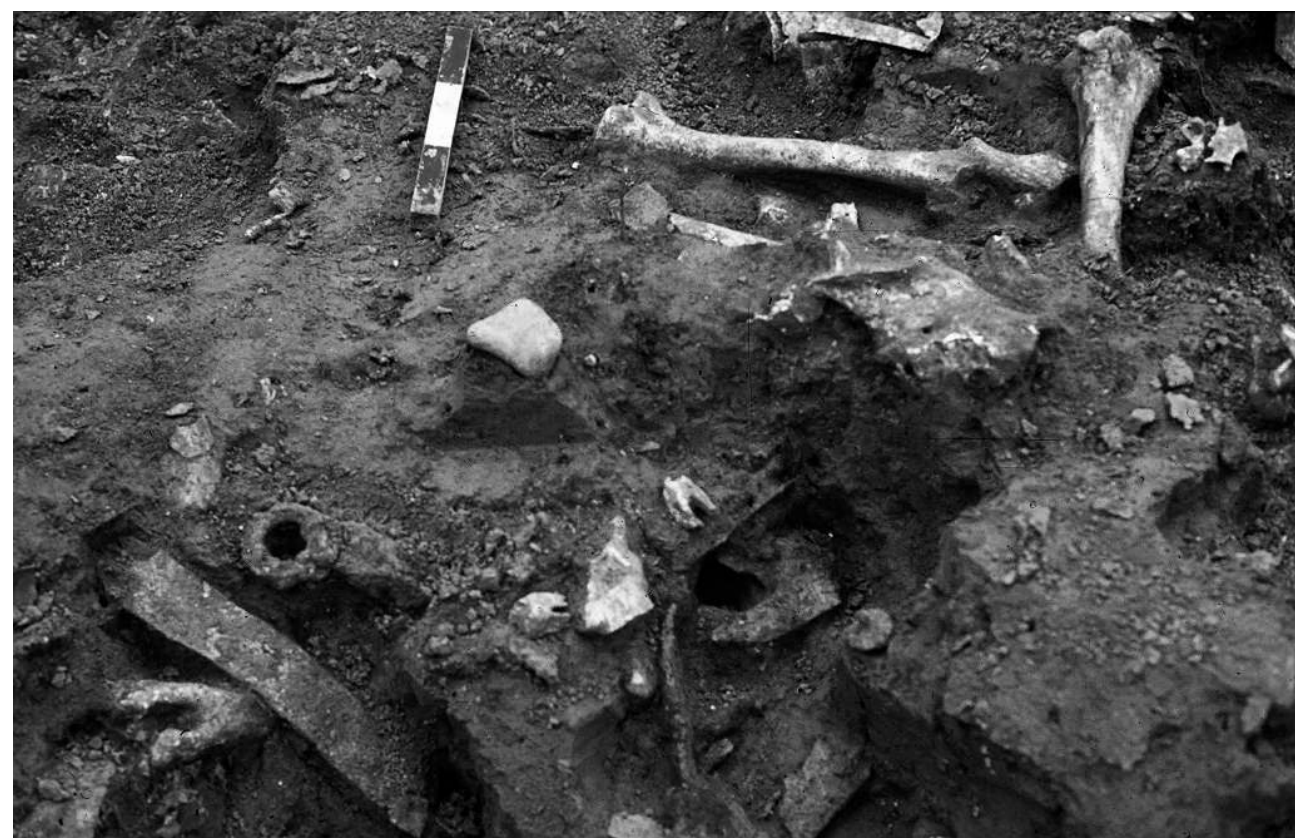

Fig. 3. Vue en cours de fouilles du fossé présentant une imbrication complexe d'ossements et d'artefacts en métal. Cl. J.-L. Brunaux.

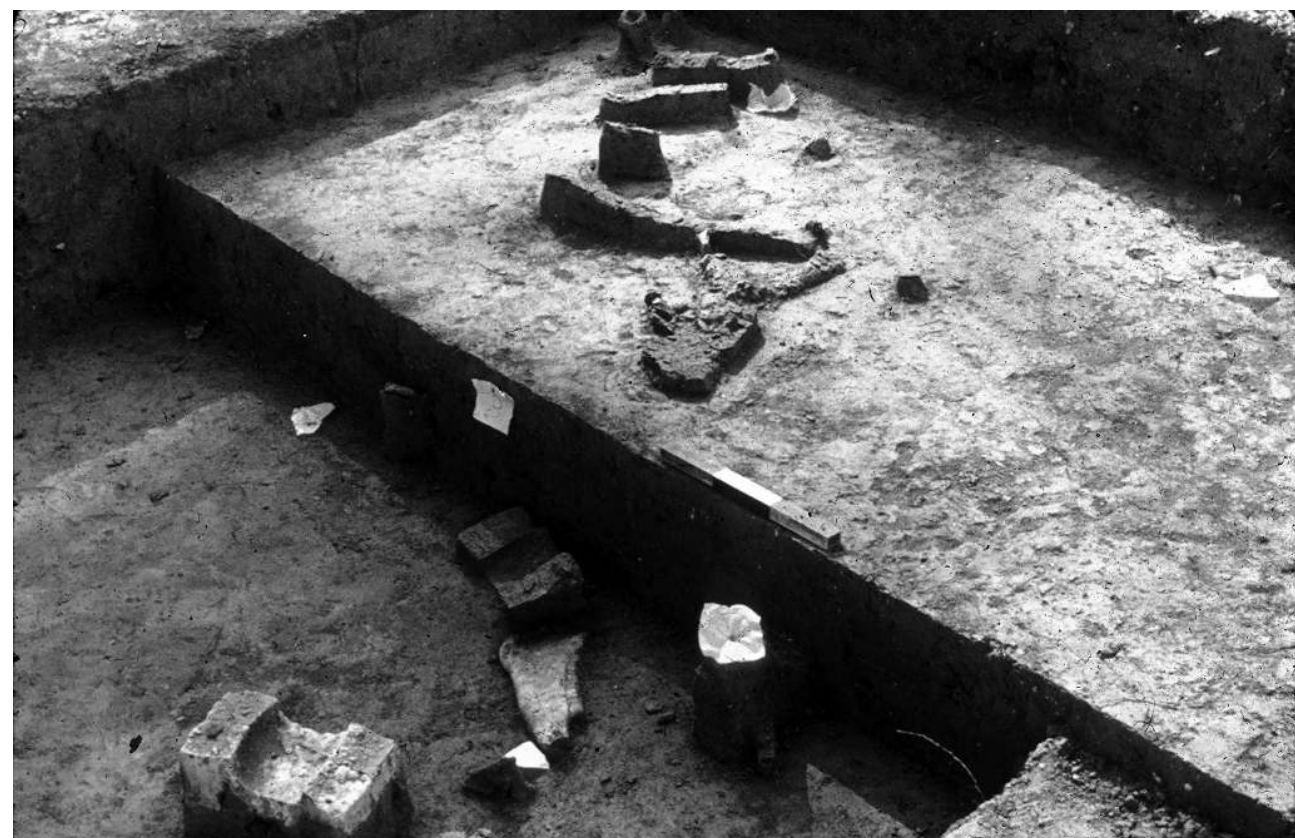

Fig. 4. Fouille du fossé par passes horizontales de 10 centimètres de profond. Cl. J.-L. Brunaux. 


\section{Déroulé des opérations archéologiques sur le sanctuaire : 1977-1982}

La première campagne de fouilles, entre mars et novembre 1977, révèle quinze mètres de fossé qui comprennent d'importants dépôts d'os et d'objets métalliques au niveau de l'entrée orientale du sanctuaire. Des sondages confirment par la suite la présence du fossé sur une longueur de quarante-cinq mètres (fig. 5).

Sont mis au jour mille cinq cents ossements en très bon état de conservation. Transportés et triés au presbytère du village mis à disposition des fouilleurs, ils font l'objet d'une étude par P. Méniel. Ce dernier met en place une méthode avec F. Poplin du Muséum National d'Histoire Naturelle, venu quelques jours sur le chantier. L'aide de ce dernier est précieuse ; il exhorte son élève à prendre en compte le moindre détail et à pousser ses observations. F. Poplin décide ensuite de s'occuper des restes humains également mis au jour, à la suite d'une observation faite sur une clavicule, qui porte les marques d'une décapitation mal effectuée.

Cette année-là, près de huit cents artefacts en fer sont sortis de terre, comprenant: des épées, leurs fourreaux et leurs systèmes de suspension, des éléments de bouclier, des armes d'hast, des fibules et quelques rares outils. Certains portent les impressionnants stigmates de mutilations volontaires. La présence exceptionnelle d'un bracelet en alliage cuivreux dans le côté sud de l'entrée fait office de marqueur chronologique, puisque son style plastique

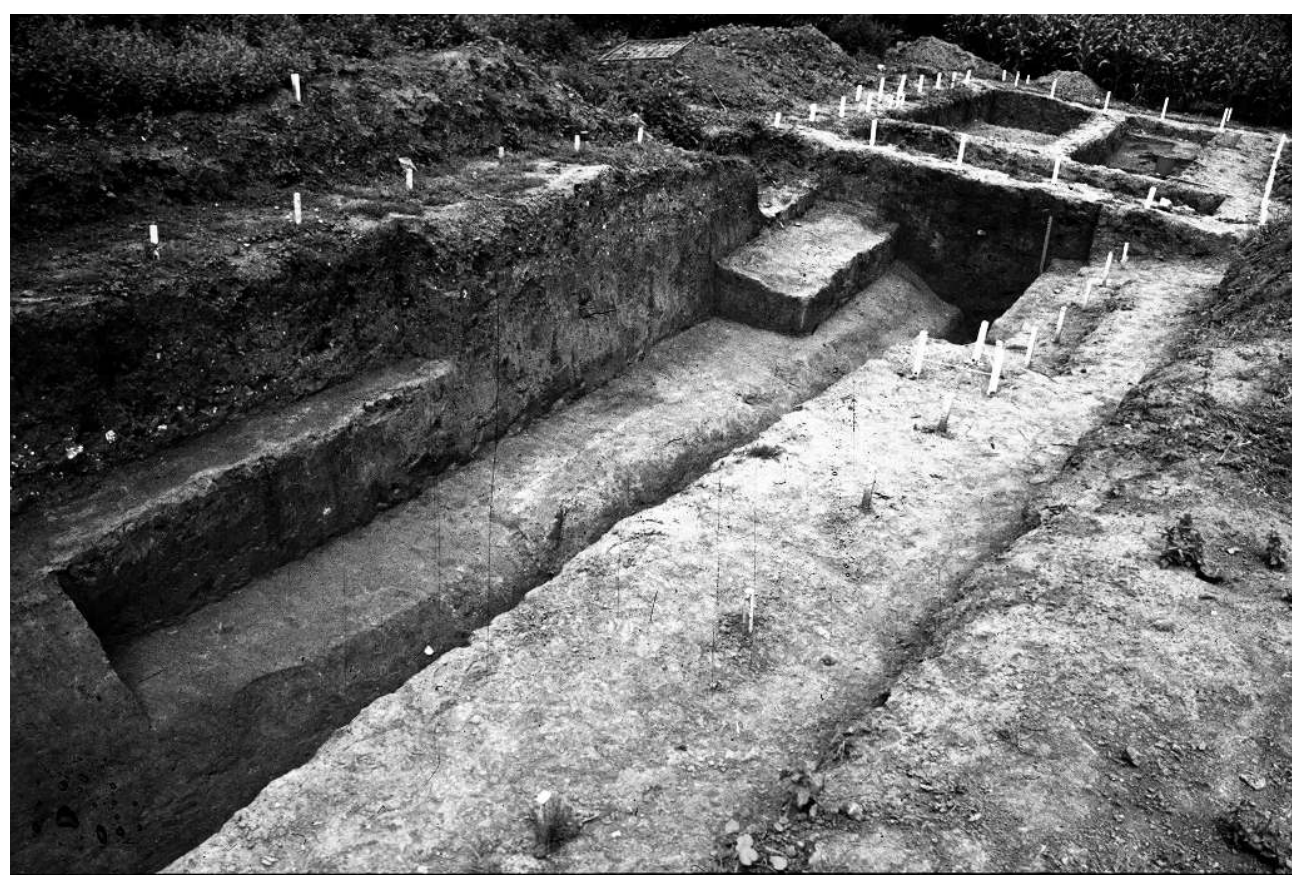

Fig. 5. Vue en cours de fouilles du côté oriental du fossé du sanctuaire. Cl. J.-L. Brunaux. 
paraît caractéristique de la seconde moitié du III siècle avant J.-C. Concernant la céramique, seules six ou sept pièces sont mises au jour. L'inventeur avance d'emblée l'hypothèse d'une structure votive, en se fondant sur plusieurs textes grecs et latins, et rejette définitivement l'existence d'une nécropole à cet emplacement.

Immédiatement, l'aspect spectaculaire des vestiges attise l'intérêt des médias et de la communauté scientifique. Pour le grand public, deux expositions temporaires sont montées, dont une itinérante, et deux reportages télévisés sont réalisés par TF1 et FR3 en 1977, sur le site et sur le travail de restauration d'urgence des artefacts à l'UTC ${ }^{19}$. Ils sont suivis de plusieurs autres documentaires et reportages, destinés à des chaînes nationales et régionales.

Les fouilles s'arrêtent à partir du mois de novembre 1977, en raison de la dégradation des conditions climatiques. Au cours de l'hiver de 1977-1978, J.-L. Brunaux explore alors l'environnement immédiat du sanctuaire, et repère un complexe défensif pouvant appartenir à un oppidum ${ }^{20}$. Le but de cette démarche est d'identifier le contexte dans lequel s'insérait le lieu de culte, et d'envisager sa place dans l'occupation du territoire. Dans le cas présent, il serait situé à l'intérieur d'une cité fortifiée, ce qui suggère le rôle communautaire qu'a pu jouer le sanctuaire. Cependant, le décalage chronologique entre les phases d'activité du lieu de culte et la date des premiers aménagements urbains semble contredire cette hypothèse.

En 1978, le reste du fossé est fouillé, à l'exception du côté ouest, masqué par le tracé d'une route départementale. L'enclos ainsi révélé, de forme quadrangulaire, couvre une superficie estiméeà mille deux cents mètres carrés. Une palissade protégeant le fossé est également mise en évidence. Le corpus ostéologique atteint cette année plus de trois mille éléments. Par l'observation des connexions anatomiques, P. Méniel identifie une stratigraphie des dépôts dans le fossé, jugée illisible auparavant (fig. 6). D'après P. Fluzin, elle est confirmée par la présence de lances entières à certains niveaux, dont les pointes et les talons sont alignés.

Cette révélation permet d'établir une chronologie relative des pratiques dans le sanctuaire. Contrairement à ce que l'on pensait l'année précédente, les milliers d'objets découverts ne furent pas déposés en une fois, mais par vagues successives, étalées dans le temps. L’idée d'un ensemble cultuel indigène, actif durant une longue période, s'affirme donc au cours de cette seconde année de fouille.

La diffusion scientifique se poursuit. Le 30 septembre 1978, une excursion sur le chantier de fouilles est organisée par le CRAVO pour les participants du

19 M. Jaussaud (dir.), Rapport d'activité du laboratoire d'étude et de restauration des métaux antiques, Direction des Antiquités de Picardie, 1978, p. 3 et p. 15.

20 J.-L. Brunaux, Rapport de 1978. 


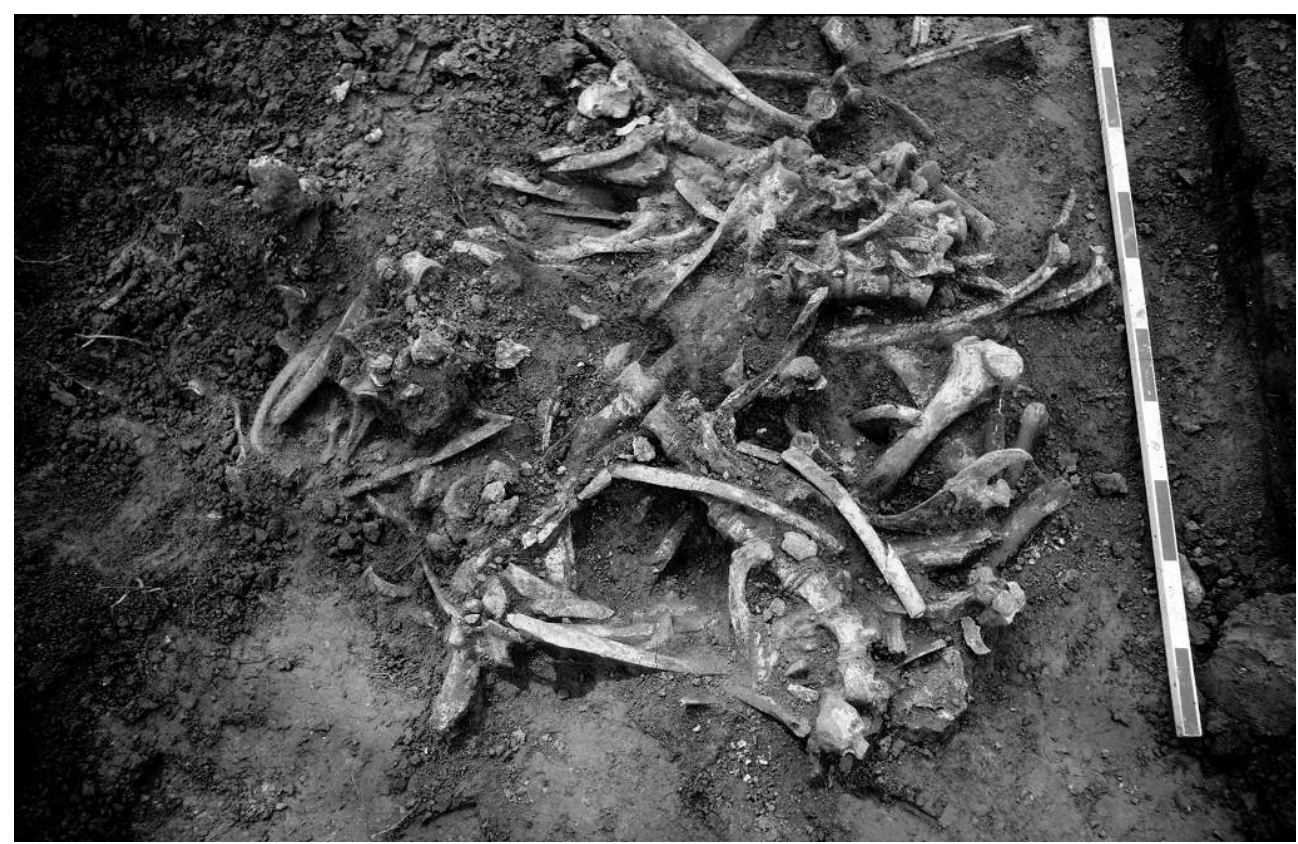

Fig. 6. Superposition d'ossements d'animaux dans les dépôts de l'entrée orientale du sanctuaire. Cl. J.-L. Brunaux.

Colloque International sur l'art celtique du Collège de France, sous la direction de P.-M. Duval et V. Kruta (fig. 7) ${ }^{21}$. Ce sont quelques-uns des plus éminents spécialistes de la Protohistoire, représentants de grandes institutions européennes, qui sont accueillis sur place. Forts de leur savoir et de leur expérience de terrain, ils ont pu conseiller et aiguiller avec précision les fouilleurs et les restaurateurs. Cette rencontre initie un mouvement de reconnaissance dans le milieu académique, rapidement consacré par les premières publications, dont une communication lors d'un colloque tenu à Tours en $1978^{22}$.

La campagne de 1979 voit la fin des opérations sur le fossé principal et fixe comme objectif la fouille de l'intérieur du sanctuaire, sur une zone d'environ huit cent mètres carrés. Le premier décapage est précédé d'une prospection géophysique menée par A. Tabbagh, venu à la demande d'O. Buchsenschutz. Cette opération révèle les structures gallo-romaines au centre de l'aire ceinte,

21 A. Rapin, “ Colloque sur l'art celtique », p. 23.

22 J.-L. Brunaux, “Un fossé à sacrifice à l'intérieur d'un retranchement dans la cité des Bellovaques ", in Travaux militaires en Gaule romaine et dans les provinces du NordOuest (colloque), Tours, Université de Tours, Caesarodunum, Bulletin de l'Institut d'Études Latines et du Centre de recherches A. Piganiol, 1978, p. 46-59. 


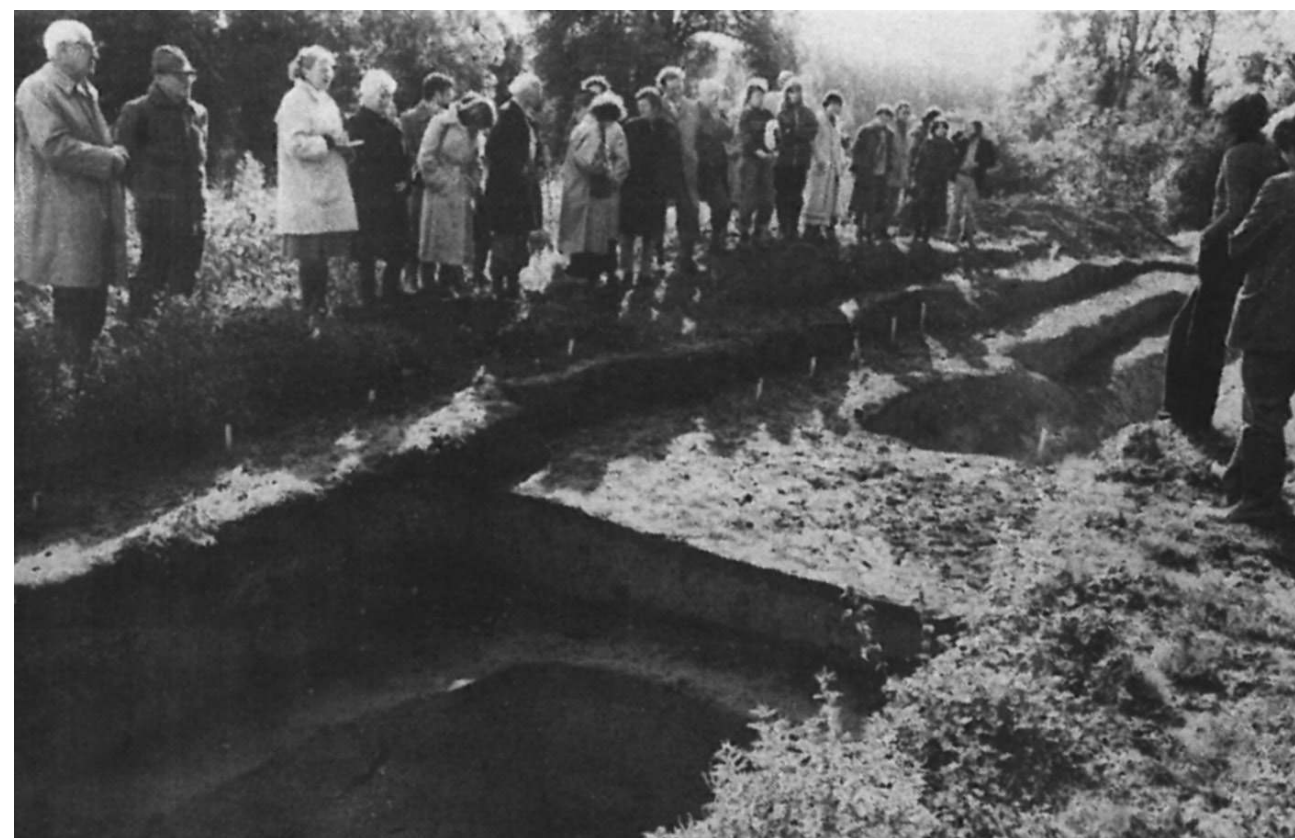

Fig. 7. Excursion des participants du Colloque International d'Archéologie Celtique du Collège de France, le 30 septembre 1978. Cl. M. Jaussaud.

construites en matériaux pérennes, dont le fanum. Ainsi, J.-L. Brunaux décide-til d'ouvrir le chantier à cet endroit, en postulant la perpétuation des lieux sacrés à travers les époques ${ }^{23}$.

Les fouilleurs sont confrontés à un enchevêtrement dense de structures, en plein centre de l'enclos. Pour développer la lecture de cet ensemble, J.-L. Brunaux choisit de prendre comme modèle l'étude menée à Libenice (République Tchèque). La publication de cet oppidum en 1962 par A. Rybova et B. Soudsky tente en effet d'expliquer l'implantation de ses structures et les concepts qu'elle présuppose (symboliques ou esthétiques notamment), grâce à la géométrie ${ }^{24}$. Cette méthode permet de mettre en évidence sept types de structures à Gournay-

23 J.-L. Brunaux, Fouille de sauvetage d'un enclos rituel de La Tène à Gournay-sur-Aronde (Oise), Rapport de fouille de sauvetage, Direction des Antiquités de Picardie, 1979, p. 2.

24 A. Ryвоva, B. Sousdky, Libenice, Kelstka Svatyne Strendnich Cechah. Sanctuaire celtique en Bohême centrale, Prague, 1962, p. 347-350. Il est à noter que la datation du site de Libenice a été réévaluée et placée à l'époque médiévale par J. Waldhauser en 1995. T. Lejars, "Historiographie de la société. Les cultes gaulois », in O. Buchsenschutz (dir.), L'Europe celtique à l'âge du Fer. VIII ${ }^{e}$ er $^{e r}$ siècles, Paris, Presses universitaires de France, collection Nouvelle Clio l'histoire et ses problèmes, 2015, p. 60. 
sur-Aronde : un habitat pré- ou protohistorique, la fosse centrale A et ses neuf fosses périphériques, trois temples successifs et le fanum. A contrario du fossé, le mobilier archéologique paraît clairsemé.

La campagne de 1979 se poursuit cette fois-ci jusqu'à l'hiver, avec des coupes réalisées au niveau du fossé d'enclos. Ces sondages mettent en évidence les structures périphériques du sanctuaire. Deux ensembles sont observés: une palissade et un fossé anciens, ainsi qu'une palissade et un fossé plus récents ${ }^{25}$. De même, la connaissance du système d'entrée se précise : une fosse en barre l'accès, ce qui suggère l'existence d'une passerelle. Une construction en bois, peut-être un porche, est également évoquée. Enfin, une tranchée est creusée de l'autre côté de la route départementale, dans l'espoir de découvrir le côté occidental du fossé ; sans succès.

Le reste de l'intérieur de l'enclos est dégagé en 1980. Plus de deux cent structures creusées dans le limon sont mises en évidence, allant du trou de piquet à la fosse, mais leur organisation demeure énigmatique. La surface fouillée ne livre que d'infimes quantités de matériel archéologique ${ }^{26}$. La dernière opération sur le sanctuaire à proprement parler concerne les prélèvements de sédiment effectués en 1982 entre la route et la parcelle, dans l'angle nord-ouest du lieu de culte. Ces interventions permettent également d'affiner la perception de la forme du fossé principal et de ses dimensions totales, mais le côté occidental semble définitivement détruit, ou situé sous la route départementale $1017^{27}$.

Les fouilles se poursuivent jusqu'en 1984 dans les environs du lieu de culte gaulois. Huit secteurs supplémentaires sont ouverts mais les structures mises au jour sont toutes postérieures ou non datées. Elles comprennent un habitat fortifié de la toute fin de l'époque gauloise indépendante, réoccupé au Haut et au Bas Empire (peut-être sous la forme d'un vicus), un quartier artisanal du Haut Empire extérieur aux fortifications, plusieurs bâtiments gallo-romains ainsi qu'un silo carolingien $^{28}$.

25 J.-L. Brunaux, Rapport de 1979, p. 8-14.

26 J.-L. Brunaux, Fouille de sauvetage d'un enclos rituel de La Tène à Gournay-sur-Aronde (Oise), Rapport de fouille de sauvetage, Direction des Antiquités de Picardie, 1980, p. 2-7.

27 J.-L. Brunaux, Fouille de sauvetage au lieu-dit «Le Parc » à Gournay-sur-Aronde (Oise), Rapport de fouille de sauvetage, Direction des Antiquités de Picardie, 1982, p. 5.

28 J.-L. Brunaux, P. MÉniel, F. Poplin, Gournay I, p. 43 -48. 


\section{Gournay-sur-Aronde : un renouvellement pluridisciplinaire ${ }^{29}$}

\section{Le fer comme document d'étude des sociétés celtiques}

À l'issue de chaque campagne de fouilles, le mobilier archéologique doit être déposé, traité et étudié pour aboutir à la diffusion des résultats. Pour Gournay-surAronde, le matériel céramique et ostéologique était transporté et stocké au Musée Antoine Vivenel de Compiègne. L'ensemble des dents humaines mises au jour avait été confié à M. Jaussaud, chirurgien-dentiste ; elles ont malheureusement disparu avec le vol de la voiture du praticien.

La gestion du matériel métallique fut davantage problématique. Face à l'ampleur de cette collection (plus de deux mille numéros d'inventaire), de sa richesse et de son contexte particulier, la nécessité de l'étudier et de la conserver dans de bonnes conditions s'impose dès 1977. Malheureusement, le laboratoire de Nancy, spécialisé dans le traitement des métaux anciens, n’a la possibilité de prendre en charge la collection que trois ans plus $\operatorname{tard}^{30}$. Sans plus attendre, P. Méniel et P. Fluzin, étudiants à l'Université Technologique de Compiègne (UTC) convainquent le directeur, G. Deniélou, de fonder un laboratoire d'urgence, associatif, nommé alors le Centre de Recherches Archéologiques et Techniques de Compiègne (CRAET).

Des locaux sont mis à disposition dans le Centre Benjamin Franklin, lié à l'UTC, en juillet 1977 pour l'installation d'un premier poste de travail (fig. 8) ${ }^{31}$. Le docteur M. Jaussaud, chirurgien-dentiste et restaurateur agréé en octobre 1977, prend la tête du laboratoire. C'est également à ce moment qu'intervient un autre acteur important de la découverte: A. Rapin. Professeur d'arts plastiques et membre du groupe archéologique du Noyonnais, il rejoint le cercle du CRAVO à sa fondation. Il participe activement à la restauration des objets de Gournaysur-Aronde, et particulièrement des armes. Son appréhension intuitive des objets lui permet de les redécouvrir sous la gangue des sédiments et de corrosion. On lui doit de grandes avancées sur la connaissance de la panoplie et par extension des modes tactiques de la guerre, composante essentielle des sociétés celtiques ${ }^{32}$.

29 À nouveau, certaines informations demeurent inédites et nous ont été communiquées oralement.

30 N. Caudron, M. Jaussaud, A. Rapin, L. Uran, "La restauration des armes gauloises provenant du dépôt de Gournay-sur-Aronde », in Revue archéologique de l'Oise, n¹3, 1978, p. 29.

31 M. Jaussaud (dir.), Rapport, p. 3.

32 Par exemple: A. Rapin, « Le ceinturon métallique et l'évolution de la panoplie celtique au III ${ }^{\mathrm{e}}$ siècle av. J.-C. », in V. Kruta (dir.), Les Celtes au III $s$. av. J. - C, Actes du IX Congrès international d'études celtiques de Paris, 8-12 juillet 1991, Paris, éditions CNRS, collection Études celtiques, 1991, p. 349-367. 


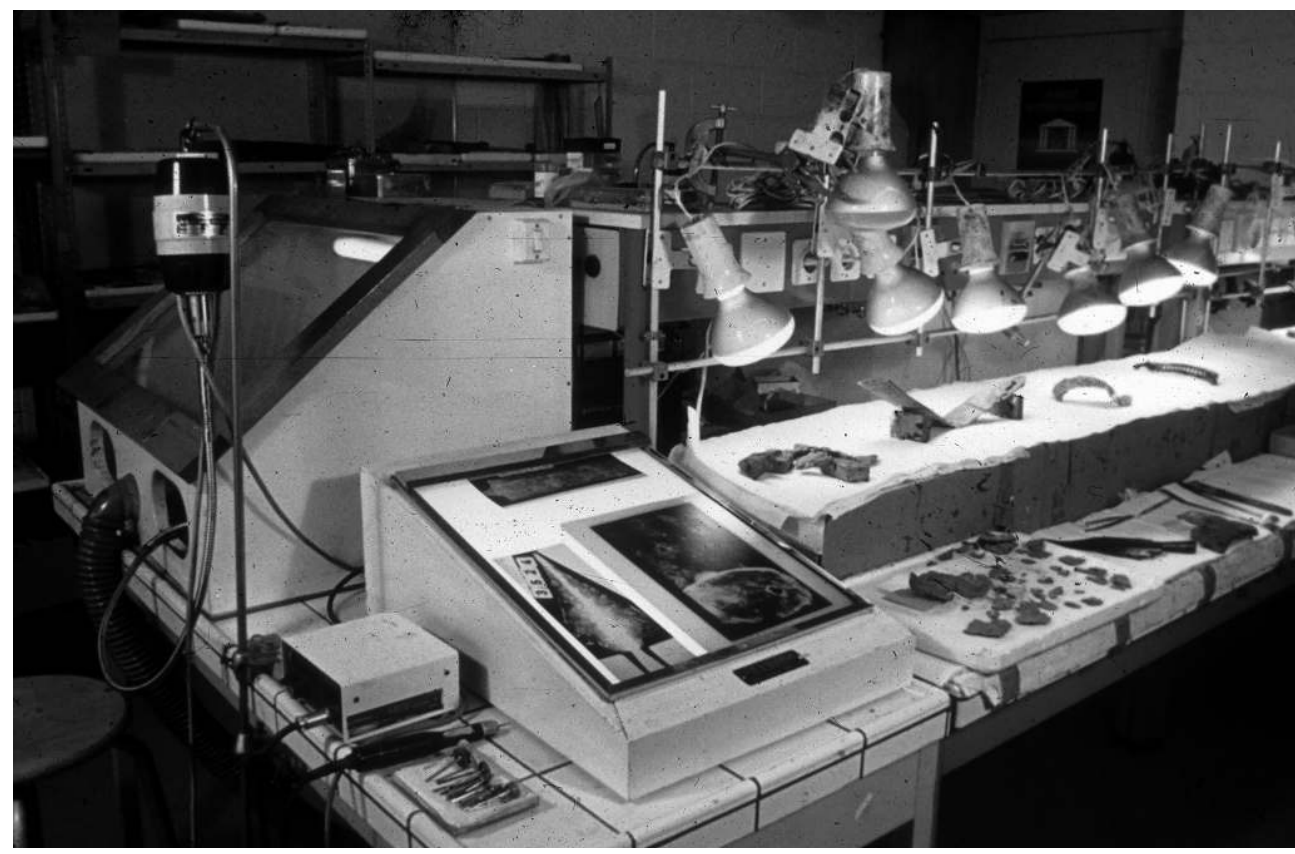

Fig. 8 : Premiers postes de restauration de l'Université Technologique de Compiègne.

Cl. J.-L. Brunaux.

Les décors apparaissent aussi, plus nombreux et complexes que ce que l'on imaginait, et amènent à réfléchir sur l'importance des armes ornées.

En 1979, le laboratoire, devenu l'Institut de Restauration et de Recherches Archéologiques et Paléométallurgiques (IRRAP), déménage dans une ancienne caserne de pompiers avec le $\mathrm{CRAVO}^{33}$. À partir de 1981, l'association se professionnalise. Elle poursuit ses activités, tout en s'adaptant à l'évolution des techniques de restauration et de stabilisation des artefacts métalliques ${ }^{34}$, jusqu'à sa fermeture définitive en 2013.

Le laboratoire se tourne très tôt vers des institutions françaises et européennes pour acquérir les savoirs techniques nécessaires, en organisant des rencontres. Par exemple, le 21 novembre 1977, A. France-Lanord, du réputé Musée du Fer de Nancy, présente une conférence à l'UTC sur les « Maladie et traitement des

33 Colnectif, La recherche archéologique en Picardie : bilans et perspectives, Actes des journées d'études d'Amiens, 21-22 mars 2005, Langres, Revue Archéologique de Picardie, n³-4, 2005, p. 299.

34 R. Bertholon, “ Nettoyage et consolidation des armes en fer du sanctuaire celtique de Gournay-sur-Aronde ", in C. Volfovsky, La conservation des métaux, Paris, Éditions CNRS, 2001, p. 103-112. 


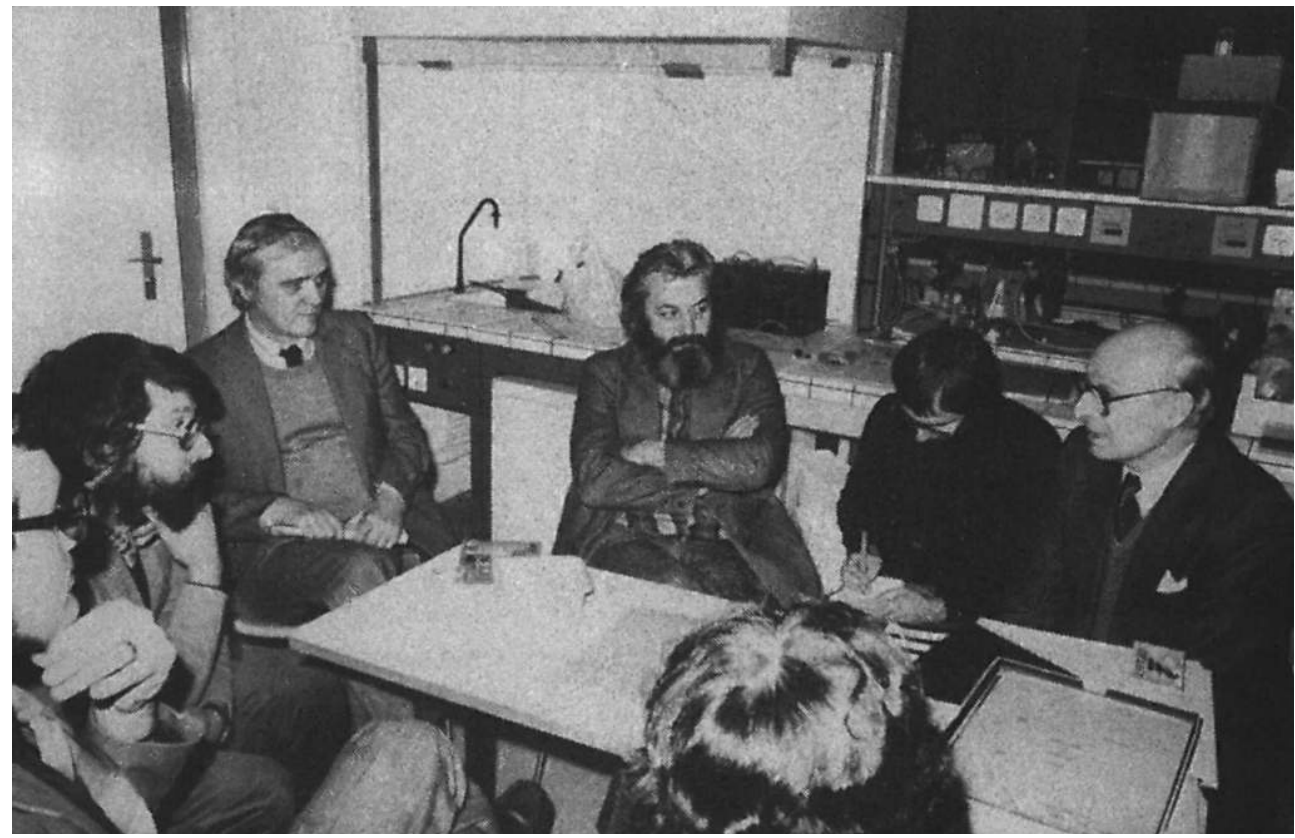

Fig. 9. Visite d'A. France-Lanord du Musée du Fer de Nancy à l'UTC, le 21 novembre 1977. Cl. M. Jaussaud.

métaux anciens ». À cette occasion, se monte une exposition impromptue d'un certain nombre d'objets de Gournay-sur-Aronde (fig. 9). En l'espace de quelques mois, l'IRRAP met au point un protocole de documentation, de nettoyage, de stabilisation et de remontage des objets avec le concours de personnalités et d'établissements importants. La conception de ce programme de restauration et de conservation a par la suite attiré de nombreuses commandes, qui ont contribué au rayonnement national de ce laboratoire ${ }^{35}$.

Du fait de son rattachement à l'UTC, l'IRRAP se fait le nouveau berceau de la paléométallurgie en France. La discipline cherche à retrouver les procédés de fabrication des artefacts en métal chez les sociétés protohistoriques, de l'extraction du minerai aux techniques de mise en forme. Grâce à la prompte ouverture de l'institut, une étude métallographique des épées de Gournay-sur-Aronde est réalisée dès 1978 par P. Méniel et L. Uran ${ }^{36}$. Par la suite, l’implantation durable

35 D'après les archives de Conservare du Musée Antoine Vivenel, Compiègne (Oise).

36 P. Meniel, L. Uran, Analyse et restauration des armes gauloises provenant du dépôt de Gournay-sur-Aronde (Oise). Premiers résultats, Université de Technologie de Compiègne, travaux de laboratoire, 1978. 
de cette discipline à l'IRRAP engendre la mise en place du premier congrès de paléométallurgie à Compiègne, les 22 et 23 février $1983^{37}$.

Ainsi, la création de l'IRRAP amorce-t-elle un attrait renouvelé pour le fer archéologique, dont le potentiel scientifique, longtemps masqué par les bubons de corrosion et les gouttelettes d'acides, se révèle enfin. Matériau de prédilection des artisans celtes, le fer se fait le support d'un programme iconographique et d'un langage formel particuliers à ces sociétés et devient légitimement un document d'étude à part entière.

\section{Du fer au rite}

L'équipe est consciente dès la première campagne de fouilles d'avoir affaire à un témoignage exceptionnel des pratiques cultuelles pré-romaines, et elle met en place un programme ambitieux d'études et de publications. La restauration exhaustive du matériel métallique - qui n'aboutira pas - et un cycle de cinq monographies sont prévus. Seulement trois paraissent; le premier opus est consacré aux structures archéologiques et à l'étude des ossements d'animaux et humains $\left(1985^{38}\right)$, les deux suivants sont dédiés aux armes en fer : les lances et les boucliers $\left(1988^{39}\right)$, puis les fourreaux d'épées $\left(1994^{40}\right)$. En parallèle, dès 1978, des articles et communications à des colloques relayent rapidement l'importance de la découverte. De plus, la richesse de la collection métallique de Gournay-surAronde aboutit à son exploitation intensive dans le cadre de nombreux travaux universitaires entre 1979 et $1993^{41}$.

Ces recherches constituent le point de départ du renouvellement des études protohistoriques françaises. Elles permettent notamment un nouvel éclairage sur les cultes celtiques, jusque-là étudiés à travers le prisme de sources indirectes : les textes antiques, grecs ou romains, l'épigraphie et l'iconographie gallo-romaine ou encore le matériel mythologique insulaire d'époque médiévale. Ces approches régressives privilégiaient l'identification d'un panthéon et l'étude du clergé druidique, mais ne donnaient pas accès aux pratiques rituelles ${ }^{42}$.

37 P. Beranger, P. Fluzin, C. Coddet, Journées de paléométallurgie, Actes du premier colloque international de l'Université de Compiègne, 22-23 février 1983, Compiègne, UTC, 1983.

38 J.-L. Brunaux, P. Méniel, F. Poplin, Gournay I.

39 J.-L. Brunaux, A. Rapin, Gournay II, Boucliers et lances, Dépôts et trophées, Paris, éditions Errance, Chevrières, Revue Archéologique de Picardie, 1988.

40 T. Lejars, Gournay III, Les fourreaux d'épée. Paris, éditions Errance. Compiègne, IRRAP, collection Archéologie aujourd'hui, 1994.

41 Par exemple : V. Merini, L'outillage agricole et artisanal et la quincaillerie du sanctuaire de Gournay-sur-Aronde, Mémoire de maîtrise sous la direction d'O. Buchsenschutz, Université de Paris I Panthéon-Sorbonne, 1990.

42 C. Quatrelivre, Le sanctuaire de Gournay. Histoire de la découverte. État de la 
La tendance s'inverse avec la découverte de Gournay-sur-Aronde. La démarche processualiste mise en œuvre consiste à retracer le fil des traitements rituels à partir des marques visibles sur les artefacts en métal et les os, de leur contexte au sein du sanctuaire et de leur état de conservation. Parmi ces pratiques, on retrouve la mise à mort des animaux, voire leur décomposition à l'air libre, l'exposition de crânes humains ou encore la destruction des armes. Ce panel de gestes codifiés a été effectué volontairement et sans but directement utilitaire, d'où leur interprétation de l'ordre du rituel, sinon du symbolique. Cependant, les croyances à l'origine de ces pratiques demeurent encore aujourd'hui difficiles à cerner.

Ces observations sur le matériel archéologique de Gournay-sur-Aronde assurent la pérennité de l'interprétation rituelle du site, qui a généré par la suite une typologie des sanctuaires picards dans la littérature ${ }^{43}$. Inévitablement, cette nouveauté amène à réévaluer d'autres hypothèses, en particulier l'interprétation rituelle des Viereckschanzen développée depuis les années 1950. Ainsi, dès 1985, J.-L. Brunaux oppose-t-il la découverte de Gournay-sur-Aronde à ces enclos quadrangulaires, principalement repérés en Allemagne du sud ${ }^{44}$, dont le caractère agricole a depuis été mis en évidence ${ }^{45}$.

Force est de constater que la démarche archéologique pour l'étude des rites se révèle particulièrement féconde. Les succès de ce renouvellement méthodologique sont introduits auprès des chercheurs antiquisants à l'occasion du colloque de Saint-Riquier en 1990, intitulé «Les sanctuaires celtiques et leurs rapports avec le monde méditerranéen ». L'événement regroupe près de deux cents chercheurs, protohistoriens et spécialistes du monde antique, notamment O. de Cazanove ${ }^{46}$,

documentation actuelle. Nouvelles analyses du mobilier métallique, Mémoire de Master 2 sous la direction de S. Verger, École Normale Supérieure de Paris, 2017, p. 11-19.

43 S. Fichtr, Les Gaulois du nord de la Gaule (150 - 20 av. J.-C.), Paris, éditions Errance, collection des Hespérides, 1994, p. 35-38.

44 J.-L. Brunaux, “Les enceintes carrées sont-elles des lieux de culte? », in O. Buchsenschutz, L. Olivier, Les Viereckschanzen et les enceintes quadrilatères en Europe celtique, Actes du IX ${ }^{\mathrm{e}}$ colloque de l'AFEAF, tenu à Chateaudun, 16-19 mai 1985, Paris, éditions Errance, collection Archéologie aujourd'hui, 1989, p. 11-14.

45 C. Von NicolaI, "La question des Viereckschanzen d'Allemagne du Sud revisitée », in I. Bertrand, A. Duval, J. Gomez de Soto, P. Maguer, Habitats et paysages ruraux en Gaule et regards sur d'autres régions du monde celtique, Actes du XXXI ${ }^{\mathrm{e}}$ colloque international de l'AFEAF, tenu à Chauvigny, 17-20 mai 2007, Chauvigny, Mémoire XXXV, 2009, p. 245.

46 O. DE Cazanove, « Ex-voto de l'Italie républicaine : sur quelques aspects de leur mise au rebut ", in J.-L. Brunaux (éd.), Les sanctuaires celtiques et leurs rapports avec le monde méditerranéen, Actes du colloque de Saint-Riquier, 8-11 novembre 1990, Paris, Éditions Errance, 1991, p. 203-214. 
J. Scheid ${ }^{47}$ et F. Jacques ${ }^{48}$. Dix ans après cette rencontre, la pertinence de l'application d'une approche archéologique pour l'histoire des religions classiques ne fait plus aucun doute. De fait, J. Scheid mentionne expressément la découverte de Gournay-sur-Aronde comme point de départ d'un tel développement ${ }^{49}$. Il s'agit d'un des rares cas d'inversion dans la hiérarchie des disciplines, lorsque la recherche en Protohistoire permet un nouvel éclairage sur l'Antiquité classique.

La collection métallique de Gournay-sur-Aronde, riche de plusieurs milliers d'objets, offre la possibilité d'élargir et d'approfondir la connaissance de la culture matérielle celtique. Les recherches sur l'armement progressent significativement : techniques de fabrication et évolutions morphologiques, mises en parallèle avec l'évolution des tactiques de guerre. Les symboles qui ornent les fourreaux et l'étude de leur langage formel font l'objet de typologies, afin de reconstruire un discours sur l'organisation sociale des Celtes. Ce développement va de pair avec les avancées de la paléométallurgie, qui permettent de détecter le choix des matériaux, les propriétés mécaniques et chimiques privilégiées par les Celtes (notamment le fer doux ${ }^{50}$, et d'identifier les procédés de mise en forme.

À l'origine de ces publications se trouve un noyau d'étudiants, de jeunes gens âgés entre dix-neuf et vingt-quatre ans. Les principaux protagonistes évoquent la découverte et la fouille du sanctuaire de Gournay-sur-Aronde à la manière d'une œuvre de jeunesse, qui a indubitablement forgé leur vocation et profondément influencé leur vie professionnelle.

Par l'expertise acquise sur le terrain et ses publications sur Gournay, J.-L. Brunaux poursuit ses recherches sur les lieux de culte celtiques et devient directeur de recherche au CNRS. Quant à P. Méniel, qui se destinait par ses études à l'UTC à l'ingénierie, il renoue avec sa passion des ossements animaux et c'est en tant qu'archéozoologue qu'il intègre le CNRS. À ce titre, il convient de souligner son rôle dans le développement de la discipline en France, en dehors du Muséum d'Histoire Naturelle. P. Fluzin, également diplômé de l'UTC, continue

47 J. Scheid, “Sanctuaires et territoire dans la Colonia Augusta Treverorum », in J.-L. Brunaux (éd.), Les sanctuaires celtiques et leurs rapports avec le monde méditerranéen. Actes du colloque de Saint-Riquier, 8-11 novembre 1990. Paris, éditions Errance, 1991, p. 42-57.

48 F. JACQUES, "Statut et fonction des conciliabula d'après les sources latines », ibidem, p. $58-65$.

49 J. ScheId, “ Pour une archéologie du rite », in Annales (Histoire, Sciences sociales), 55, 3, 2000 , p. 616.

50 L. Uran, “ Observations métallographiques sur les épées celtiques en fer », in A. Duval, J. Gomez De Soto (éd.), Sur les âges du Fer en France non méditerranéenne, Actes du VIII ${ }^{\mathrm{e}}$ colloque de l'AFEAF, tenu à Angoulême, 18-20 mai 1984, Bordeaux, Aquitania, supplément 1, 1986, p. 299-308. 
sur la voie de la paléométallurgie avec ses premières expérimentations en 1981. Il intègre également le CNRS et fonde plusieurs unités de recherche successives dans son domaine, dont l'IRAMAT en 1999.

Bien que submergée par ses milliers d'artefacts, l'équipe a su lire les gestes rituels qui ont abouti à leur dépôt dans les fossés de Gournay-sur-Aronde. Cette approche représente une première dans l'histoire de la discipline, annonçant le renouvellement durable de l'étude des cultes celtiques par les vestiges archéologiques.

Le travail effectué sur le sanctuaire gaulois a été remarquable, si l'on tient compte de la jeunesse des fouilleurs, des aléas de l'archéologie de l'époque et des ressources limitées de l'opération. Véritable laboratoire méthodologique, la fouille et l'étude du site ont parfois été soumises à l'adaptation, voire à l'improvisation de moyens. Malgré ces difficultés, l'équipe a conduit ce sanctuaire à la postérité en l'érigeant en corpus de référence.

Ce succès répond à la fois à l'attention accrue portée au patrimoine en Picardie dans les années 1970, mais aussi à l'aspiration des inventeurs du site à mener des études pluridisciplinaires, à publier exhaustivement et à analyser sous toutes ses coutures un ensemble qu'ils savent exceptionnel. C'est également dans ce contexte que naît le creuset d'innovations qu'est l'IRRAP à sa création en 1977 .

Pourtant, Gournay-sur-Aronde demeure profondément méconnu du grand public et très partiellement publié pour les spécialistes. Il est apparu que seulement 30,7\% de la collection avait fait l'objet d'une publication ou d'un travail universitaire, à l'issue de la reprise d'étude du mobilier archéologique en $2015^{51}$. Ainsi, quarante ans après cette découverte, l'exploitation future des données inédites voit le jour dans le cadre d'un projet collaboratif de recherche. Tirant profit d'une rare qualité d'enregistrement et des importants travaux de nos prédécesseurs, ce programme aspire à se placer dans la continuité des inventeurs de Gournay-sur-Aronde. Il sera enfin temps de présenter à l'ensemble de la communauté un site d'exception, dans toute sa complexité et sa diversité.

\author{
Carole Quatrelivre \\ Doctorante \\ École Normale Supérieure \\ UMR 8546 AOrOc \\ PSL Université Paris \\ 3 rue Henri Martin \\ 94200 Ivry-sur-Seine. \\ carole.quatrelivre@ens.fr
}

51 C. Quatrelivre, Le sanctuaire de Gournay. Histoire de la découverte, p. 142. 
ANABASES

Traditions et Réceptions de l'Antiquité

Revue de l'équipe de recherche E.R.A.S.M.E.

Université Toulouse-Jean Jaurès (UT2J)

NoRMES RÉDACTIONNELLES

ANABASES publie des articles dans cinq langues : français, anglais, allemand, italien et espagnol.

Les articles ne dépasseront pas 35000 signes et seront conformes aux normes de la revue,

disponibles sur le site web : http://plh.univ-tlse2.fr

Les articles seront pourvus d'un résumé en français et en anglais, ainsi que de six à huit mots-clés dans ces deux langues.

Les articles pourront être accompagnés de planches en noir et blanc.

Les comptes rendus compteront de 4500 à 6000 signes.

Site web avec Présentation, Sommaire de tous les Numéros et Bulletin d’Abonnement

http://plh.univ-tlse2.fr

Revues.org : http://anabases.revues.org

\section{Courrier}

Pour les articles :

Clément Bur (clement.bur@univ-jfc.fr)

Catherine VALENTI (catherine.valenti@univ-tlse2.fr)

Pour les comptes rendus :

NoémieVILlacÈQue (noemie.villaceque@univ-reims.fr)

Université Toulouse-Jean Jaurès (UT2J)

Équipe P.L.H. - E.R.A.S.M.E. (EA4601)

Maison de la recherche (MdR)

5, allées Antonio Machado

F-31058 Toulouse Cedex 9

Tél. : 0033/(0)5.61.50.25.56 et 57

Fax : 0033/(0)5.61.50.24.90 


\section{Historiographie et identités culturelles}

Carole QuATRELIVRe, Le sanctuaire gaulois de Gournay-sur-Aronde (Oise). Retour sur une découverte exceptionnelle des années 1970

\section{Traditions du patrimoine antique}

Christophe CorbIER, Le pindarisme et l'archéologie musicale : style, valeur et authenticité de la première Pythique à l'époque moderne

Arnaud AmiLien, Hélène en Égypte : Hérodote en dialogue avec l'épopée

\section{Archéologie des savoirs}

Sébastien CAzalas, Au jardin des exempla. Rhétorique et stratégie de l'exemplum antique dans l'œuvre politique de Jean Juvénal des Ursins (1388-1473)

Dossier - Perpétuer Ovide : aspects moraux, éditoriaux, linguistiques et culturels $\left(\mathrm{xIV}^{\mathrm{e}}-\mathrm{XvIII}{ }^{\mathrm{e}} \mathrm{s}\right.$.)

Francesca DelL'Oro, Introduction

Hélène Casanova-Robin, L'audace châtiée : Phaéton, Actéon et Icare dans la tradition latine jusqu'à la Renaissance, tours et détours d'un symbolisme

Dylan Bovet, Le commentaire latin des Métamorphoses d'Ovide : pratiques humanistes et évolutions de Regius-Micyllus (1543) à Burmann-Heinsius (I727)

Martine Furno, Ovide en classe, ou un auteur en éclats

Basil Neurs, D'un Ovide chrétien à un Ovide burlesque, du Moyen Âge au Grand Siècle : continuités et changements dans la traduction et dans l'illustration des Métamorphoses perçus à travers deux éditions du xvir siècle

Olivier ThÉvenaz, Épilogue

\section{Actualités et débats}

Tiphaine-Annabelle BESNARD, 2019 : l'année pop des musées d'archéologie. Retour sur l'exposition romaine Il classico si fa pop. Di scavi, copie e altri pasticci

\section{Relire les classiques des sciences de l'Antiquité}

Jean-Pierre AıBERT, Le premier Detienne : une relecture de « La notion mythique d'A $\lambda \hat{\eta} \theta \varepsilon\llcorner\alpha$ » (REG, 1960, p. 27-35)

Marcel DetIENNE, La notion mythique d'A $\lambda \hat{\eta} \theta \varepsilon \varepsilon \alpha$

\section{L'atelier de l'histoire : chantiers historiographiques}

Antiquités numériques (coordonné par Élodie Guillon) (1)

Élodie GuILLON, Introduction

Jaime ALvar, Le projet EPIDI : Epítetos divinos. Experiencia religiosay relaciones de poder en Hispania

Les mots de l'Antiquité (coordonné par Magali Soulatges) (10)

Jack Tномаs, L’Antiquité dans les toponymes de l’État de New York

Actualité du théâtre (coordonné par Malika Bastin-Hammou) (4)

Mathieu Ferrand, “Avons-nous perdu le Soleil ? / Ou l'avons-nous chassé ? ”

Thyeste de Sénèque, traduit par Florence Dupont. Mise en scène de

Thomas Jolly (Avignon, 2018).

Voyages et Voyageurs (coordonné parVéronique Krings) (11)

Claude Azıza, Freud à Pompéi

\section{Comptes rendus de lecture}

